\title{
Reduction of the $\mathrm{Yb}$ valence in $\mathrm{YbAl}_{3}$ nanoparticles
}

\author{
D. P. Rojas, L. Fernández Barquín, J. I. Espeso, and J. Rodríguez Fernández \\ CITIMAC, Facultad de Ciencias, Universidad de Cantabria, 39005 Santander, Spain
}

\begin{abstract}
J. Chaboy
Instituto de Ciencia de Materiales de Aragón and Departamento de Física de la Materia Condensada, CSIC-Universidad de Zaragoza, 50009 Zaragoza, Spain
\end{abstract}

(Received 29 November 2007; revised manuscript received 23 July 2008; published 16 September 2008)

\begin{abstract}
Measurements of specific heat, dc magnetic susceptibility, and $\mathrm{Yb} L_{\mathrm{II}}$ and $L_{\mathrm{III}} \mathrm{x}$-ray absorption near-edge structure (XANES) and extended $\mathrm{x}$-ray absorption fine structure (EXAFS) on $\mathrm{YbAl}_{3}$ milled alloys are reported. $\mathrm{X}$-ray diffraction patterns are consistent with a reduction in particle size down to $10 \mathrm{~nm}$ and an increase in the lattice strain up to $0.4 \%$ for $120 \mathrm{~h}$ of milling time. A decrease in the mean valence from 2.86 for the unmilled alloy to 2.70 for $120 \mathrm{~h}$ milled $\mathrm{YbAl}_{3}$ is obtained from the analysis of XANES spectra. From the analysis of spectra in the EXAFS region, an increase in the mean-square disorder of neighbor distance with milling time is detected in good agreement with the results of x-ray diffraction. Size effects strongly influence the magnetic and thermal properties. The value for the maximum of the magnetic susceptibility decreases around $30 \%$ for $120 \mathrm{~h}$ milled alloy and an excess specific heat, with a peak around $40 \mathrm{~K}$ in the milled samples, is derived. These changes in the physical properties along the milled $\mathrm{YbAl}_{3}$ alloys are associated with the reduction in particle size. Such a reduction leads to the existence of a large number of $\mathrm{Yb}^{2+}$ atoms at the surface with respect to the bulk affecting the overall electronic state.
\end{abstract}

DOI: 10.1103/PhysRevB.78.094412

PACS number(s): 75.30.Mb, 65.40.Ba, 75.40.-s

\section{INTRODUCTION}

Magnetic fine particle systems are subject of interest attracting much scientific activities today because of the variety of interesting phenomena arising from the existence of nanometric structures. ${ }^{1,2}$ Within this area of research, the vast majority of the studies have been devoted to systems comprising transition metals. ${ }^{3}$ However, little attention has been paid to nanocrystalline rare-earth intermetallics. Among these, several studies have been reported which are related to the $\mathrm{GdX}_{2}$ Laves phases under mechanical milling. In this case, the relationship between the structural and magnetic changes induced by the milling process results in the evolution from a ferromagnetic behavior to a spin-glass phase, which emerges at lower temperatures. ${ }^{4-8}$ More recently, a study of the magnetic properties of $\mathrm{TbAl}_{2}$ milled alloys has revealed that not only disorder but also size effects are important in crucial understanding of the magnetic behavior, with a pronounced variation of coercivity with the grain size. ${ }^{9}$

The relevance of the extension of these studies of nanocrystalline particle systems to $\mathrm{Ce}$ and $\mathrm{Yb}$ intermetallics, the latter being known as strongly correlated electron materials, requires some understanding of how macroscopic properties in these systems become modified at the nanoscale. In this sense, results have been reported in Ce-Al (Ref. 10) and Ce-Pt (Ref. 11) systems over recent years (with samples formed by nanoparticles of average size of 8 and $4 \mathrm{~nm}$, respectively). The samples were produced in a liquid-nitrogen cold trap by flash evaporation of bulk ingots. The experimental results in $\mathrm{CeAl}_{2}, \mathrm{Ce}_{3} \mathrm{Al}$, and $\mathrm{Ce}_{3} \mathrm{Al}_{11}$ intermetallics showed a crossover from magnetic ordering to an enhanced Kondo behavior when the particle size was reduced. ${ }^{10}$ In addition, extended $\mathrm{x}$-ray absorption fine structure (EXAFS) measurements performed in $\mathrm{CeAl}_{2}$ and $\mathrm{CePt}_{2}$ indicate that size effects on the electronic state of these nanosized alloys must also be considered. ${ }^{11}$ Very recently, there have been reportings of a decrease in antiferromagnetic correlations and an enhancement of Kondo interactions with the reduction in the size of the particles in nanosized $\mathrm{CePt}_{2}(3.1-26 \mathrm{~nm})$. This has been suggested by measurements of specific heat and magnetic susceptibility. ${ }^{12}$ No particular correlation between the electronic properties and the large surface contribution in particles of such a small size is discussed in those recent reports on the Ce alloys.

Specific theoretical and experimental data about nanoparticles of strongly correlated materials are scarce. In this sense, the interplay between Ruderman-Kittel-KasuyaYoshida interaction and Kondo effect in nanosized systems is still not well understood despite recent efforts on this issue. $^{13,14}$ Alternatively, it could be fruitful to take into account the previous work on the variations of the electronic properties due to surface effects in rare-earth systems and to relate it to experimental evidence. ${ }^{15}$

In order to shed some light on this matter, a comprehensive study of the series of $\mathrm{YbAl}_{3}$ milled alloys has been undertaken in the present work. For this we have selected $\mathrm{YbAl}_{3}$, which has been thoroughly studied in the past due to its intermediate-valence (IV) properties, as a starting compound to analyze the downsizing effects on the electronic properties. This intermetallic presents a broad maximum around $100 \mathrm{~K}$ in the magnetic susceptibility and in the magnetic contribution to specific heat. ${ }^{16-20} \mathrm{~A}$ crossover slower than predicted by the Anderson impurity model from the low-temperature Fermi-liquid regime to the high-temperature local-moment regime was also observed. ${ }^{17}$ Moreover, anomalies appearing in the transport and magnetic properties at low temperatures were attributed to coherence effects, which are suppressed by the magnetic field and chemical disorder. ${ }^{19}$ In addition to these exciting properties, $\mathrm{Yb}^{3+}$ ion 
presents the mirror electronic configuration with respect to $\mathrm{Ce}^{3+}$ and provides complementary information especially when different parameters are tuned typically by diluting and/or applying pressure. ${ }^{21}$ This wealth of details enables us to address the changes on the magnetic, thermal, and electronic properties of an archetypal IV alloy of $\mathrm{YbAl}_{3}$ when the particle size is reduced in a controlled manner by a milling process and eventually to correlate those modifications with structural and/or disorder modifications.

\section{EXPERIMENT}

A starting polycrystalline $\mathrm{YbAl}_{3}$ pellet was prepared by arc melting suitable amounts of pure constituents $\mathrm{Yb}(3 \mathrm{~N})$, $\mathrm{Al}(5 \mathrm{~N})$ (Alfa) in an arc furnace under protective $\mathrm{Ar}$ atmosphere and was subsequently annealed at $750{ }^{\circ} \mathrm{C}$ for five days. Equally, a $\mathrm{LuAl}_{3}$ alloy was also prepared as a nonmagnetic isostructural counterpart to appraise the magnetic contribution to the specific heat. The analysis of the x-ray diffraction pattern is consistent with a cubic structure of the type $\mathrm{AuCu}_{3}$ (space-group $P m \overline{3} m$ ) with a unit-cell parameter $a=4.1918(4) \AA$ for $\mathrm{LuAl}_{3}$ and 4.2057(7) $\AA$ for $\mathrm{YbAl}_{3}$, which are in excellent agreement with previously reported data. ${ }^{22}$ The reduction in the particle size of bulk $\mathrm{YbAl}_{3}$ was carried out through mechanical milling, which is considered a practical route to obtain large quantities of nanocrystalline materials. ${ }^{23}$ Amounts around $5 \mathrm{~g}$ of bulk $\mathrm{YbAl}_{3}$ were crushed and then milled in a planetary high-energy ball milling system (Retsch PM 400/2) at a rotation speed of $200 \mathrm{rpm}$. A small amount of milled samples was collected at 20,70, and $120 \mathrm{~h}$ of milling time. In order to avoid oxidation, handling and storage were carried out in a glove box under argon atmosphere.

The initial structural characterization was carried out in a Philips PW 1710 diffractometer with $\mathrm{Cu} K_{\alpha}$ radiation. The crystallographic parameters were determined using a Rietveld powder profile program ${ }^{24}$ with silicon as a standard to account for the instrumental broadening. The macroscopic thermal and magnetic properties were collected in a Quantum Design Physical Properties Measurement System device in the temperature range of 2-300 K.

$\mathrm{X}$-ray absorption near-edge structure (XANES) and EXAFS measurements were performed at the beamline BL39XU of SPRING-8 facility and beamline BM25A of ESRF. All the spectra were recorded in the transmission mode using a Si double-crystal monochromator and gasionization chambers as detectors. A single layer of the fine powder was put onto a kapton tape and the appropriate sample thickness was obtained by stacking multiple layers of the tapes for the measurements. In addition, an $\mathrm{Yb}_{2} \mathrm{O}_{3}$ (99.998\%, Alfa) compound was used as a reference for the $\mathrm{Yb}^{3+}$ contribution.

\section{RESULTS}

\section{A. X-ray diffraction}

Figure 1 shows X-ray diffraction patterns for the series of $\mathrm{YbAl}_{3}$ milled alloys. A progressive broadening and reduction in the intensity of the peaks, commonly related to both a

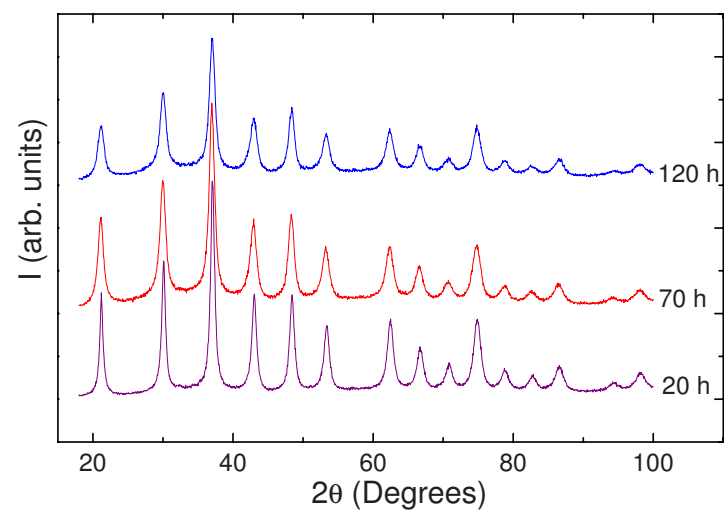

FIG. 1. (Color online) X-ray diffraction patterns for the $\mathrm{YbAl}_{3}$ milled alloys at 20,70, and $120 \mathrm{~h}$ of grinding time. The spectra have been shifted up for clarity. The peak broadening is especially evident for the sample milled during $120 \mathrm{~h}$.

decrease in the particle size $(D)$ and an increase in the lattice strain $(\eta)$, is observed. ${ }^{9}$ The Rietveld refinement of the $\mathrm{x}$-ray diffraction data shows a decrease in the particle size down to $10 \mathrm{~nm}$ and an increase in the lattice strain up to $0.4 \%$ for 120 $\mathrm{h}$ of milling time. The refinement was carried out using the $P m \overline{3} m$ space group and the Thompson-Cox-Hastings pseudo-Voigt function for the peak profile, similar to that employed in the analysis of $\mathrm{TbAl}_{2}$ milled alloys. ${ }^{9}$ The size of the particles and the lattice strain as function of the milling time for the $\mathrm{YbAl}_{3}$ series is depicted in Fig. 2. It is interesting to note that the strain here is not so strong as the ones reported for other milled rare-earth intermetallics such as $\mathrm{GdAl}_{2}$ (Ref. 4) or $\mathrm{TbAl}_{2},{ }^{9}$ where $\eta$ reaches a value around $1 \%$. Interestingly enough, the lattice parameter does not change along the series, ruling out severe lattice distortions; presumably pressure effects associated with changes in the unit-cell volume will not have a large influence on the physical properties.

\section{B. dc magnetic susceptibility}

Measurements of dc magnetic susceptibility $\chi(T)=M / H$ have been carried out at $H=1 \mathrm{~T}$ for the series of milled

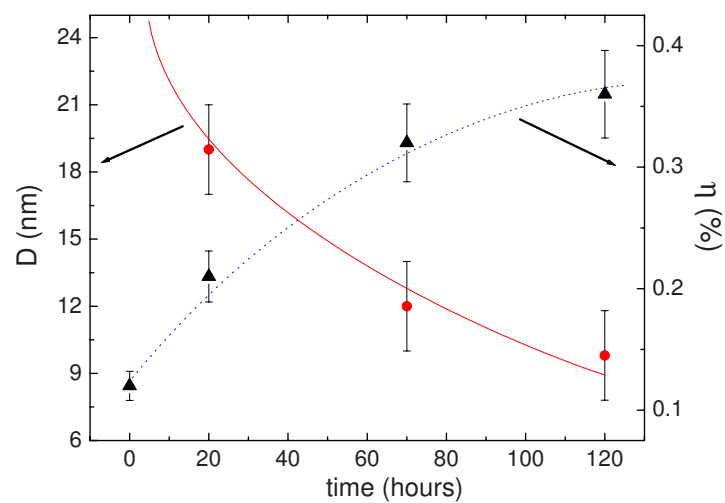

FIG. 2. (Color online) Particle size $(D)$ and strain $(\eta)$ as functions of the milling time for the $\mathrm{YbAl}_{3}$ milled alloys. The decrease in size and increase in strain are features usually found in milled metallic systems. Continuous and dashed lines are guides for the eye. 


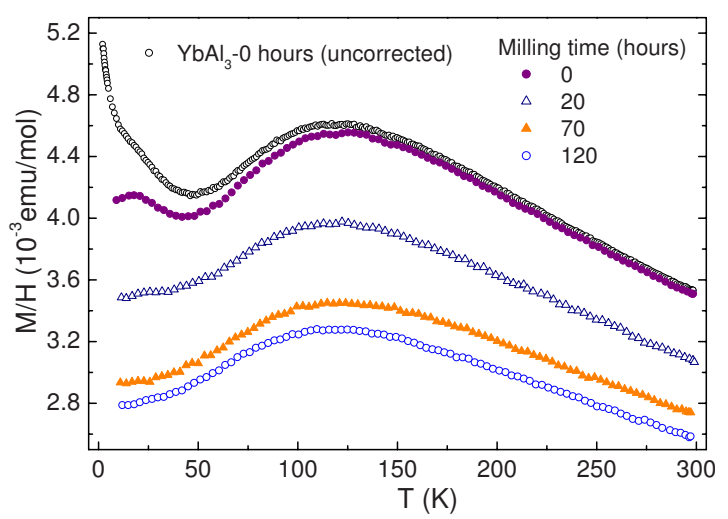

FIG. 3. (Color online) Temperature dependence of dc magnetic susceptibility $\chi(T)$ at $H=1 \mathrm{~T}$. The maximum around $121 \mathrm{~K}$ for the unmilled alloy decreases slightly in the position but decreases strongly in absolute value with the increase in milling time. A lowtemperature anomaly around $T=17 \mathrm{~K}$ associated with coherence effects is clearly observed in the unmilled sample and is hardly present in the alloy milled for $20 \mathrm{~h}$.

$\mathrm{YbAl}_{3}$ samples. A maximum characteristic of IV systems is observed in all the curves. However, below $50 \mathrm{~K}$, a contribution arising from $\mathrm{Yb}_{2} \mathrm{O}_{3}$ was also detected. The presence of $\mathrm{Yb}_{2} \mathrm{O}_{3}$ has been commonly reported in $\mathrm{YbAl}_{3}$ alloys, appearing even in single crystals. ${ }^{16}$ This contribution should be subtracted to obtain the intrinsic magnetic susceptibility of the samples. In order to perform this subtraction, we have measured the magnetic susceptibility of $\mathrm{Yb}_{2} \mathrm{O}_{3}$ (Alfa, 99.998\%). The weight percentages of this oxide obtained from the fit of the susceptibility at low temperatures are rather small, i.e, $0.4 \%, 1.9 \%, 2.7 \%$, and $3.4 \%$ for the unmilled, 20, 70, and $120 \mathrm{~h}$ milled samples, respectively. In spite of the use of an inert atmosphere during the milling process, the proportion of the $\mathrm{Yb}$ oxide clearly increases with the milling time. This is no surprise considering that the size of the particles is reduced below $10 \mathrm{~nm}$ (see Fig. 2) and, hence, the surface/volume fraction becomes very large, favoring the oxidation process. In Fig. 3 we show the temperature dependence of the magnetic susceptibility of $\mathrm{YbAl}_{3}$ samples at different milling times after the correction to the contribution of $\mathrm{Yb}_{2} \mathrm{O}_{3}$ and, as commented above, a broad maximum appears in all the samples. For comparison purposes, the uncorrected data of unmilled alloy are also presented, showing a characteristic upturn of the $\mathrm{Yb}_{2} \mathrm{O}_{3}$ impurity, which is more evident for $T<50 \mathrm{~K}$. This sample presents two maxima around 121 and $17 \mathrm{~K}$ as already reported for this material. ${ }^{16-19}$ The low-temperature maximum is associated with lattice coherence effects being very sensitive to disorder and magnetic field and revealing a good quality of the sample. ${ }^{17}$ This maximum is virtually absent in the $20 \mathrm{~h}$ milled alloy and practically disappears for $120 \mathrm{~h}$ of milling time samples (probably as a result of the disorder increase), which is induced by the milling process. ${ }^{4-9} \mathrm{Re}-$ garding the high-temperature maximum (usually associated with the Kondo temperature in these IV systems), ${ }^{18}$ there is a slight decrease $(1 \%-2 \%)$ in the position directly affected by the mentioned Curie-Weiss behavior of the $\mathrm{Yb}_{2} \mathrm{O}_{3}$. However, the absolute value of the magnetic susceptibility decreases

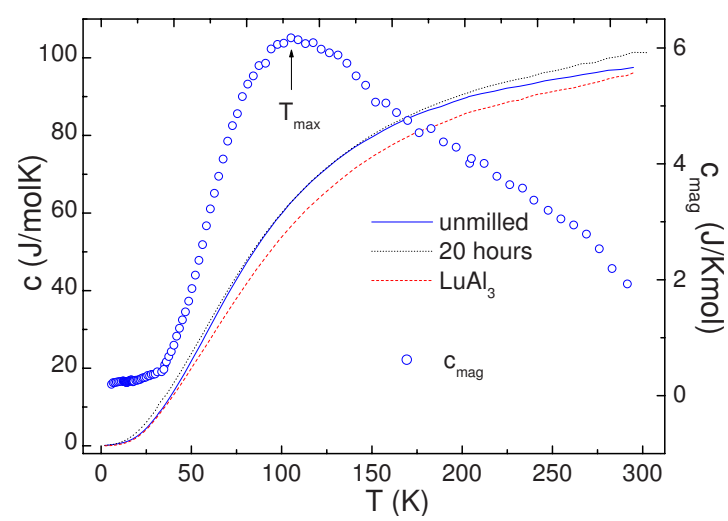

FIG. 4. (Color online) Temperature dependence of the specific heat for unmilled and $20 \mathrm{~h}$ milled alloys. An excess of the specific heat is observed around $40 \mathrm{~K}$ and above $160 \mathrm{~K}$ for the milled sample. The dashed line is the specific heat of $\mathrm{LuAl}_{3}$. A maximum in $c_{\text {mag }}$ of the unmilled alloy appears around $100 \mathrm{~K}$.

$14 \%, 25 \%$, and $30 \%$ for 20,70 , and $120 \mathrm{~h}$ of milling time, respectively, which is a significant sign of an increasing presence of $\mathrm{Yb}^{2+}$ contribution.

\section{Specific heat}

Specific-heat measurements can provide useful information about the electronic, phonon, and magnetic behaviors of the $\mathrm{YbAl}_{3}$ milled samples. In Fig. 4 the experimental temperature dependence of the specific heat of unmilled, $20 \mathrm{~h}$ milled $\mathrm{YbAl}_{3}$, and bulk $\mathrm{LuAl}_{3}$ (nonmagnetic counterpart) alloys is presented. For the unmilled alloy, the extraction of the magnetic contribution $\left(c_{\mathrm{mag}}\right)$ is straightforward by subtracting the specific heat of the nonmagnetic counterpart $\mathrm{LuAl}_{3}$ alloy as shown in Fig. 4. A maximum at around $100 \mathrm{~K}$ is found to be in good agreement with reported results for this material. ${ }^{17-19}$ However, there are salient features of the milled alloy with respect to the unmilled one, which require an alternative analysis: (i) an increase in slope above $160 \mathrm{~K}$ and (ii) an excess of specific heat around $40 \mathrm{~K}$. These two contributions in the specific heat for the milled samples are found in nanocrystalline materials as established experimentally ${ }^{25-27}$ and explained theoretically. ${ }^{28-31} \mathrm{~A}$ procedure to better determine such modifications should bear in mind that fine particle materials are frequently formed by a collection of grains, which are separated by an interface. The atomic fraction of the interface component is estimated to be $3 \delta / D$, where $\delta$ is the thickness of the grain boundary [typically $3-4$ atomic layers $(\approx 1 \mathrm{~nm})]$ and $D$ is the average crystallite size. ${ }^{25}$ Thus, for our nanosized $\mathrm{YbAl}_{3}$ alloys, about $16 \%, 25 \%$, and $30 \%$ of the atoms are on the interface for the $20 \mathrm{~h}, 70 \mathrm{~h}$, and $120 \mathrm{~h}$ milled samples, respectively. Since the physical environment of the atoms at the interface is different from those of atoms located inside the crystallites, the macroscopic properties would be affected by the particle size reduction. In this sense, changes in the configuration of nearest neighbors (loss of coordination of the atoms at the interface) will have a strong influence on the thermodynamic and magnetic properties. Indeed, in nanocrystalline $\mathrm{YbAl}_{3}$ milled samples, the dc magnetic-susceptibility results are strongly 


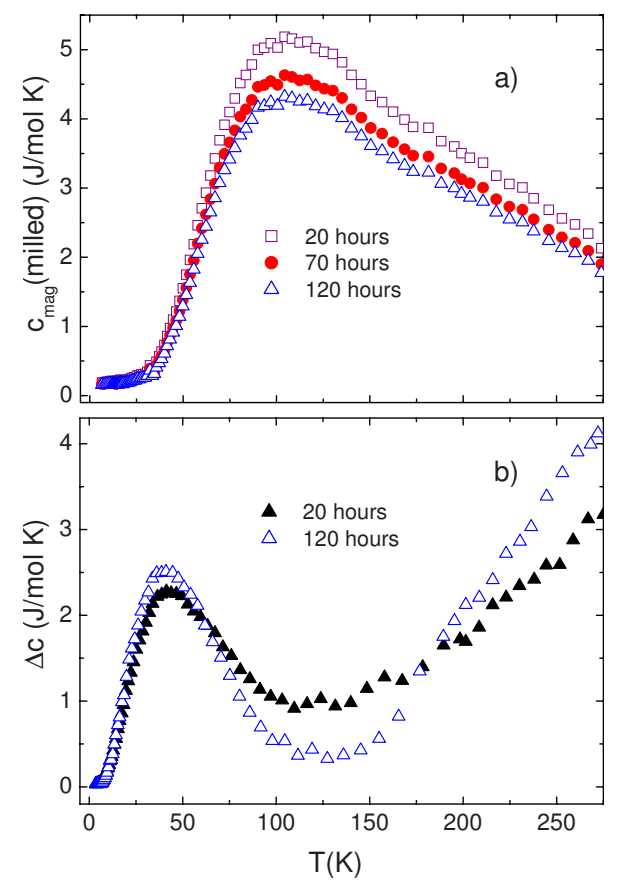

FIG. 5. (Color online) (a) Magnetic contribution to the specific heat vs temperature curves calculated for the series of $\mathrm{YbAl}_{3}$ milled alloys $\left[c_{\text {mag }}(\right.$ milled $)=\omega c_{\text {mag }}($ bulk $)$, where $\omega$ is the fraction of the $\mathrm{Yb}$ atoms in the valence state of the bulk]. (b) Excess specific heat for the $\mathrm{YbAl}_{3}$ milled alloys. A peak around $40 \mathrm{~K}$ and a slope above 160 $\mathrm{K}$ (increasing with the milling time) are observed (see text).

affected by the milling process (grain-size decrease) as described in Sec. III B. An intrinsic origin for the reduction in the absolute value of dc magnetic susceptibility while decreasing in size is related precisely to those atoms lying at the surface of the nanoparticles. It can be assumed that the electronic state is $\mathrm{Yb}^{2+}$ for such surface atoms in analogy to what occurs on the surface of both rare-earth films ${ }^{32,33}$ and bulk materials. ${ }^{34}$ Hence, to appraise the magnetic contribution $\left[c_{\text {mag }}(\right.$ milled $\left.)\right]$ to the specific heat, it is necessary to take into account such a $\mathrm{Yb}^{2+}$ influence. Therefore, such a magnetic contribution [see Fig. 5(a)] can be estimated as $c_{\text {mag }}($ milled $)=\omega c_{\text {mag }}$ (bulk), where $\omega$ is the fraction of the $\mathrm{Yb}$ atoms in the valence state of the bulk. The values of $\omega$ are then $84 \%(20 \mathrm{~h}), 75 \%(70 \mathrm{~h})$, and $70 \%(120 \mathrm{~h})$. We presume that the specific heat of milled (nanosized) $\mathrm{YbAl}_{3}$ alloys can be expressed in the form $c$ (milled $)=c_{\text {mag }}$ (milled) $+c\left(\mathrm{LuAl}_{3}\right.$ unmilled $)+\Delta c$, where $\Delta c$ is the excess specific heat due to the contribution of the interface in the nanosized alloy. In such a simple equation we know $c$ (milled) and $c\left(\mathrm{LuAl}_{3}\right.$ unmilled) experimentally and consequently the excess specific heat $\Delta c$ has been extracted and plotted in Fig. 5(b). The $\Delta c(T)$ curves display a maximum around $40 \mathrm{~K}$ and a slope increase above $160 \mathrm{~K}$. The excess specific heat increases, as expected, with the reduction in the particle size. This tendency is similar to that observed not only in nanometer-sized crystalline $\mathrm{Cu}$ and $\mathrm{Pd}$ between 150 and 300 $\mathrm{K}$, where the specific-heat values were higher than those in the polycrystalline state, ${ }^{25}$ but also in nanocrystalline $\mathrm{Ni}_{80} \mathrm{P}_{20}$ with particles between 5 and $10 \mathrm{~nm}$-showing an enhanced specific heat with a maximum at about $40 \mathrm{~K} .{ }^{27}$ Computer



FIG. 6. (Color online) $\mathrm{Yb} \mathrm{L}_{\mathrm{III}}$ normalized $\mathrm{x}$-ray absorption $\left(\mu_{\text {Norm }}\right)$ vs relative photon energy $E-E_{0}\left(E_{0}=8946.5 \mathrm{eV}\right.$ is the energy of the maximum of the first derivative of the spectrum of the reference $\mathrm{Yb}_{2} \mathrm{O}_{3}$ sample) for the unmilled, 20, 70, and $120 \mathrm{~h}$ milled $\mathrm{YbAl}_{3}$ and $\mathrm{Yb}_{2} \mathrm{O}_{3}$ reference sample. A change in the $\mathrm{Yb}^{2+}$ contribution is clearly observed (marked with an arrow) and is also shown in detail in the inset.

simulations of a nanocrystalline model material also predict the existence of a low-temperature specific-heat peak associated with low-frequency phonon modes. ${ }^{30}$ It is easy to assume that the reduction in the number of nearest neighbors in the interface results in a decrease in the density of the system. Considering this fact and based on a quasiharmonic Debye approximation, Wagner ${ }^{29}$ offered a good description of the low-temperature anomaly and the linear increase in the high-temperature region ( $T>150 \mathrm{~K}$ in our case), which is in reasonable agreement with the experimental results in nanocrystalline $\mathrm{Pd}$.

In order to provide a microscopic point of view for the origin of the observed presence of an $\mathrm{Yb}^{2+}$ contribution in the magnetic susceptibility and the existence of an excess specific-heat stemming from interfacial atoms, we present the results on the electronic properties and the short-range structure as sampled by X-ray absorption measurements, XANES, and EXAFS.

\section{D. $L_{\mathrm{II}}$ and $L_{\mathrm{III}} \mathrm{x}$-ray absorption}

XANES spectroscopy is a sensitive probe for the electronic structure of a given element in any material. ${ }^{35} \mathrm{Yb}_{\mathrm{II}^{-}}$ and $L_{\mathrm{III}}$-edge XANES measurements were carried out on the milled $\mathrm{YbAl}_{3}$ alloys and $\mathrm{Yb}_{2} \mathrm{O}_{3}$ reference sample. In Fig. 6, the results for unmilled, $120 \mathrm{~h}$ milled $\mathrm{YbAl}_{3}$, and $\mathrm{Yb}_{2} \mathrm{O}_{3}$ at the $L_{\mathrm{III}}$ edge are displayed. For comparison purposes the data were normalized after a pre-edge background subtraction by fitting with a linear function and by using a relative scale of energy $E-E_{0}$, where $E_{0}=8946.5 \mathrm{eV}$ is the energy of the maximum of the first derivative of the spectrum of the reference $\mathrm{Yb}_{2} \mathrm{O}_{3}$ sample. A clear rise in $\mathrm{Yb}^{2+}$ contribution around $-5 \mathrm{eV}$ was observed, corresponding to the energy of $8941 \mathrm{eV}$ for the $L_{\mathrm{III}}$ edge with the increase in the milling time, which is also replicated in the results at the $L_{\mathrm{II}}$ edge (not shown). Subsequently, the valence of $\mathrm{Yb}$ was deter- 
mined from the obtained XANES spectra following the procedure similar to that used for the analysis of $\mathrm{LuAl}_{3}$ and $\mathrm{YbAl}_{3}$ alloys. ${ }^{18,36}$ In those results, the $\mathrm{LuAl}_{3}$ spectrum was used to account for the $\mathrm{Yb}^{3+}$ contribution while the sum of an arctangent function representing the edge absorption and a Lorentzian function representing the white line were considered for the divalent $\mathrm{Yb}^{2+}$ contribution. Then, the XANES spectra can be considered as

$$
\mu(E)=n_{f} \mu_{3+}(E)+\left(1-n_{f}\right) \mu_{2+}(E),
$$

where $n_{f}$ is the $f$-occupation number, $\mu_{3+}$ is the contribution from $\mathrm{LuAl}_{3}$, and $\mu_{2+}$ is the $\mathrm{Yb}^{2+}$ divalent contribution. In the fitting of $\mathrm{YbAl}_{3}$ series of milled alloys, we used the result obtained for the spectrum of $\mathrm{LuAl}_{3}$ by Lawrence et al. ${ }^{36}$ The obtained values for the valence $(\nu)$ (where $\nu=2+n_{f}$ ) are 2.86, 2.75, 2.71, and 2.71 for unmilled, 20, 70, and $120 \mathrm{~h}$ milled alloys, respectively.

To establish the influence of the $\mathrm{Yb}_{2} \mathrm{O}_{3}$ impurity phase in the valence obtained from XANES, a simple approximation is to consider that each oxide molecule possesses two $\mathrm{Yb}^{3+}$ ions, then leading to

$$
\nu=\left(\nu_{\exp }-6 y\right) /(1-2 y),
$$

where $y$ stands for the concentration of the $\mathrm{Yb}_{2} \mathrm{O}_{3}$ impurity phase. This procedure has already been successfully used in the case of $\mathrm{YbCu}_{5-x} \mathrm{Ga}_{x}$ series. ${ }^{37}$ In our case we inserted the impurity concentration values previously extracted from the dc magnetic susceptibility in Sec. III B. Thus, after correction, the modified values for $\nu$ are 2.86, 2.74, 2.70, and 2.70 for unmilled, 20, 70, and $120 \mathrm{~h}$ milled alloys, respectively. In any case, the changes with respect to the uncorrected values are smaller than $1 \%$. Thus, the main result is a diminishing value of the valence with the milling process with an abrupt decrease between the unmilled and $20 \mathrm{~h}$ milled alloys.

Measurements of the X-ray absorption coefficient $(\mu)$ for photon energies above the absorption edge in the extended region (EXAFS) are extremely useful to probe the nearneighbor environment of a particular atomic species in a material. ${ }^{35}$ In order to extract the EXAFS oscillations from the absorption curve, the experimental data of the $\mathrm{YbAl}_{3}$ series of milled alloys were analyzed with the IFEFFIT program as implemented in the ATHENA and ARTEMIS packages. ${ }^{38}$ The background subtraction was performed using the AUTOBK algorithm, which determines an empirical background spline based on a distinction between data and background in terms of Fourier components. The edge-step normalization of the data was determined by a linear preedge subtraction and the regression of a quadratic polynomial beyond the edge. The difference between these two polynomials extrapolated to the edge energy $E_{0}$ is used as the normalization constant resulting in the EXAFS function $\chi(k)=\left[\mu(k)-\mu_{0}(k)\right] / \mu_{0}(k)$, where $k$ is the photoelectron wave vector. The result of this procedure is shown in Fig. 7 for the unmilled sample (raw data), which also depicts the EXAFS filtered functions and their fits (solid lines) for all samples. There is no significant difference between unmilled and nanosized $\mathrm{YbAl}_{3}$ alloys up to $8 \AA^{-1}$ in the region where the oscillation amplitudes are larger. Above $8 \AA^{-1}$ the small oscillations are at similar positions, reflecting an expected

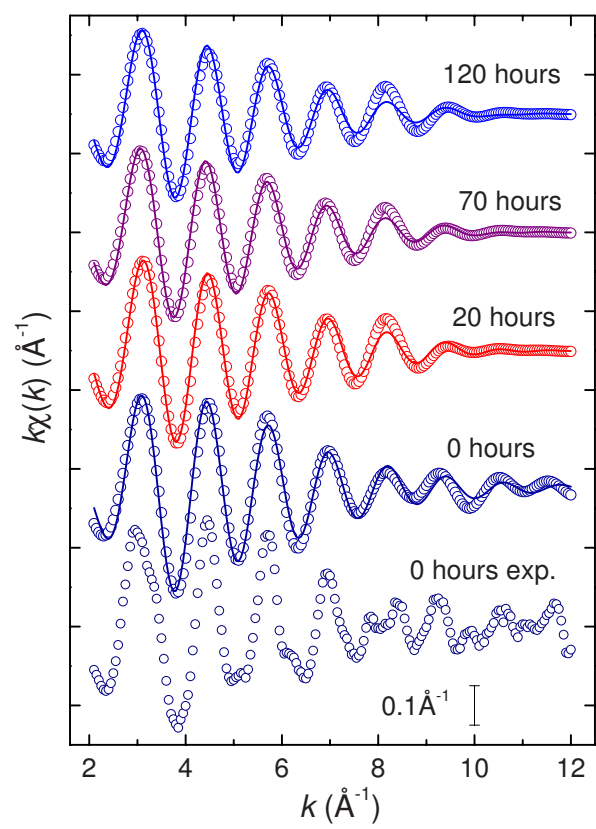

FIG. 7. (Color online) Fourier filtered EXAFS functions $\chi(k) k$ weighted and their fits (solid lines) as a function of photoelectron wave vector $k$ for unmilled and 20,70, and $120 \mathrm{~h}$ milled $\mathrm{YbAl}_{3}$ alloys. The fits were restricted to first shell in the range $k$ $=2-12 \AA^{-1}$ with $r=1.1-3.2 \AA$ using a Hanning window function. For the unmilled sample the experimental data are also shown.

overdamping in the nanosized alloys with respect to the bulk sample. ${ }^{11}$

The Fourier transform of $\chi(k)$ gives a series of peaks in $r$ space corresponding to each shell of neighbor atoms as shown in Fig. 8. $\mathrm{YbAl}_{3}$ crystallizes in the cubic $\mathrm{AuCu}_{3}$ structure (space-group $P m \overline{3} m$ ) with a lattice parameter $a$ $=4.2057(7) \AA$ as commented above. In this structure Yb atoms occupy the Wyckoff position $1 a$, surrounded in the first coordination shell by $12 \mathrm{Al}$ atoms at a distance of $2.9739 \AA$ and a second-neighbor shell with $6 \mathrm{Yb}$ atoms at a distance of $4.2057 \AA$. Thus, the main peak in Fig. 8 is assigned to the first shell of $\mathrm{Yb}$-Al correlations. Furthermore, the Yb-edge spectra were fitted with a nonlinear least-squares routine

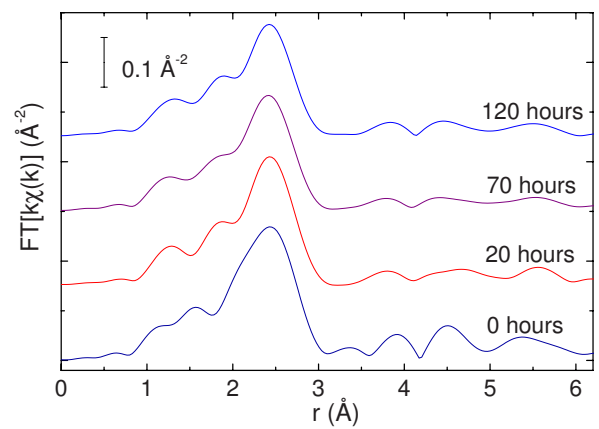

FIG. 8. (Color online) Amplitude of the $k$-weighted Fourier transform in $r$ space taken at $L_{\mathrm{III}}$ edge for unmilled, 20, 70, and 120 $\mathrm{h}$ milled $\mathrm{YbAl}_{3}$ alloys. $\mathrm{Yb}-L_{\mathrm{III}}$-edge data are transformed from $k$ $=2-12 \AA^{-1}$ using a Hanning window function. The main peak correspond to the contribution of the first shell of nearest neighbors (12 $\mathrm{Al}$ atoms). 
TABLE I. Results of the fit to the first shell of EXAFS $k$-weighted functions in $\mathrm{YbAl}_{3}$ milled alloys. $r$ is the radial distance to the nearest neighbor and $\sigma^{2}$ is the Debye-Waller factor. $R$ factor: standard value to measure the goodness of the fit. A single value for the amplitude reduction factor $S_{0}^{2}=0.7$ and for the coordination number $N=12$ (nominal value) were used for all the samples. $S_{0}^{2}$ was determined from the fit of the unmilled $\mathrm{YbAl}_{3}$ alloy.

\begin{tabular}{cccc}
\hline \hline $\begin{array}{c}\text { Milling time } \\
\text { (hours) }\end{array}$ & $\begin{array}{c}r \\
(\AA)\end{array}$ & $\begin{array}{c}\sigma^{2} \\
\left(\AA^{2}\right)\end{array}$ & $R$ factor \\
\hline 0 & $2.96(1)$ & $0.0069(22)$ & 0.045 \\
20 & $2.96(2)$ & $0.0088(17)$ & 0.037 \\
70 & $2.96(2)$ & $0.0105(17)$ & 0.034 \\
120 & $2.96(3)$ & $0.0115(21)$ & 0.051 \\
\hline \hline
\end{tabular}

ARTEMIS. ${ }^{38}$ The analysis was restricted to the first shell in the range $k=2-12 \AA^{-1}$ with $r=1.1-3.2 \AA$ using a Hanning window function. The free variables used during the fitting were a common energy origin shift, interatomic distance $(r)$, and Debye-Waller factor $\left(\sigma^{2}\right)$ for the first shell. For the value of the many-body amplitude reduction factor, $S_{0}^{2}$ was usedthe value being obtained from analyzing the $\mathrm{YbAl}_{3}$ unmilled sample spectrum. The coordination number $(N)$ was fixed to the nominal value of 12-corresponding to the first shell of nearest-neighbors $\mathrm{Al}$ atoms. The numerical results of the fitting procedure are reported in Table I. The $r(\AA)$ values indicate that the interatomic distances for the milled samples are not affected by the milling process (see Fig. 8), whereas the Debye-Waller factor increases when the milling time increases. This last feature is consistent with the rise in the lattice strain calculated by the Rietveld refinement of the $\mathrm{x}$-ray diffraction data as depicted in Fig. 9. In addition, the values of $\sigma^{2}$ are of the order of those reported for $\mathrm{Co}-\mathrm{Cu}$ granular nanometric alloys, ${ }^{39}$ in nanogranular $\mathrm{Ni} / \mathrm{NiO}$ system, ${ }^{40}$ and smaller than those of amorphous rare-earth systems. $^{41}$

\section{DISCUSSION}

Changes of the valence on $\mathrm{Yb}$ IV materials have been promoted usually by applying pressure ${ }^{42}$ and (or) by chemi-

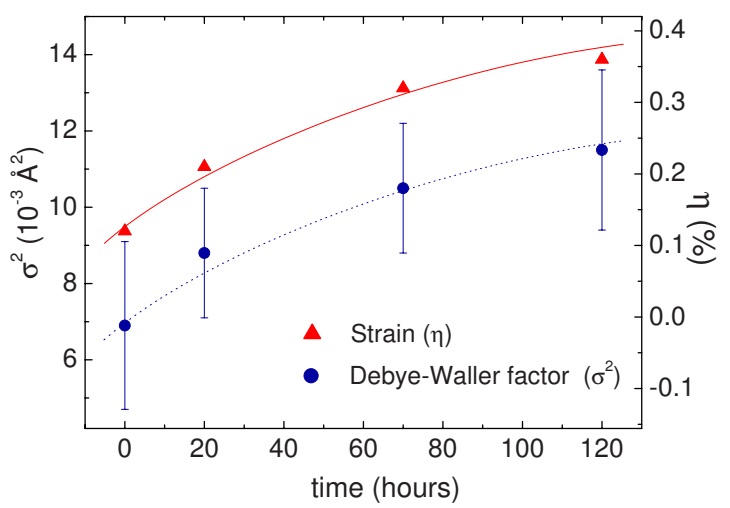

FIG. 9. (Color online) Lattice strain $(\eta)$ and Debye-Waller factor $\left(\sigma^{2}\right)$ in the unmilled, 20, 70 and $120 \mathrm{~h}$ milled alloys as a function of the milling time. The lines are guides for the eye.

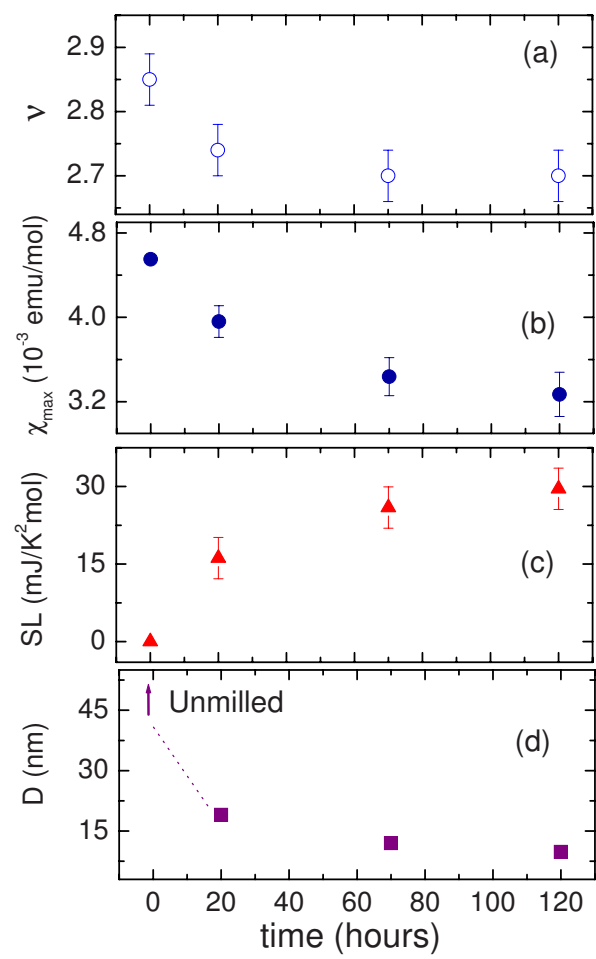

FIG. 10. (Color online) Milling time dependence of the valence $\nu$ (a), the value of the magnetic susceptibility at maximum $\left(\chi_{\max }\right)$ (b), Slope of the high-temperature excess contribution to the specific heat $(S L)(\mathrm{c})$, the size of the particles $(D)(\mathrm{d})$. The grain size of the unmilled alloy can only be suggested (marked with an arrow). Errors in $D$ are smaller than the square symbol.

cal substitution as reported in $\mathrm{Yb}_{1-x} \mathrm{Lu}_{x} \mathrm{Al}_{3},{ }^{18} \mathrm{YbCu}_{5-x} \mathrm{Ga}_{x}{ }^{37}$ and $\mathrm{Yb}_{1-x} \mathrm{M}_{x} \mathrm{Al}_{2}(M=\mathrm{Ca}, \mathrm{Sc})$ series ${ }^{43}$ to cite a few examples. The pressure dependence is the result of a balance between the gain in cohesive energy for the $\mathrm{Yb}^{3+}$ configuration (demonstrated by the smaller atomic volume) and the energy price to promote a $4 f$ electron into the conduction band. For instance, the dependence of valence on applied pressure has been studied in the IV material $\mathrm{YbAl}_{2}$ by resonant inelastic $\mathrm{X}$-ray emission with the result that the $\mathrm{Yb}$ valence increases from 2.25 at ambient pressure to 2.9 at $385 \mathrm{kbar}^{44}$ On the other hand, XANES results for the valence variation at 300 $\mathrm{K}$ in the chemically substituted $\mathrm{Yb}_{1-x} \mathrm{Lu}_{x} \mathrm{Al}_{3}$ series reveal a decrease from $\nu=2.8$ for $\mathrm{YbAl}_{3}$ to 2.7 for $\mathrm{Yb}_{0.1} \mathrm{Lu}_{0.9} \mathrm{Al}_{3}{ }^{18}$ Our results in the study of $\mathrm{YbAl}_{3}$ series of milled alloys show that the milling process also induces variations in the electronic state of this IV material. The analysis of XANES results at $300 \mathrm{~K}$ is consistent with a change of the valence from 2.86(3) for the unmilled alloy to 2.70 (3) for $120 \mathrm{~h}$ of milling time as shown in Fig. 10(a). The milling process contributes to the production of nanoparticles, which modifies the magnetic and thermal properties. Very recent studies on other correlated electron nanosized materials such as $\mathrm{CeAl}_{2}, \mathrm{Ce}_{3} \mathrm{Al}, \mathrm{Ce}_{3} \mathrm{Al}_{11}$, and $\mathrm{CePt}_{2}$ have revealed the influence of size effects on the electronic state and a crossover from magnetic ordering to an enhanced Kondo behavior. ${ }^{10-12}$ This is somewhat connected to the study of the influence of the sign and strength of the size-induced spin polarization of host conduction-band electrons of single Fe impurities embedded in $\mathrm{Cu}$ and $\mathrm{Ag}$ nanoparticles. ${ }^{45}$ 
In the present case, the results show a weak modification in the susceptibility maxima $(1 \%-2 \%)$, which is not as significant as the change of its absolute value (30\%). This change is a clear indication of the presence of an increasing fraction of $\mathrm{Yb}^{2+}$ atoms with the increase in the milling time. Hence, our milled alloys are constituted by a collection of nanoparticles with two types of atoms; those in the core present an IV state (as in the bulk) whereas those sitting on the surface and forming part of a probably distorted interface (as revealed by the specific-heat measurements) display a $\mathrm{Yb}^{2+}$ valence due to the variation of the coordination number. ${ }^{15,32}$ In some ways this grain arrangement is similar to that reported for nanosized $\mathrm{TbAl}_{2}$ (Ref. 9) and $\mathrm{GdAl}_{2}$ (Refs. 7 and 8 ) (for the case of $4 f$ systems) and many other systems involving $3 d$ alloys. $^{3}$ Other reports on $\mathrm{YbAl}_{3}$ single crystals $^{46}$ cannot be brought forward for the analysis of our system, although it is striking for future work that the existence of magnetic clusters is discussed.

Apart from the pure size effects, it is necessary to discuss the disorder created by the milling process. Besides the reduction in the particle size, an increase in the lattice strain is observed (see Fig. 9). From x-ray diffraction data an increase in the lattice strain up to $0.4 \%$ is obtained for $120 \mathrm{~h}$ milled alloy. Therefore, the disorder here is not so strong as reported in other series of milled rare-earth intermetallics such as $\mathrm{GdAl}_{2}$ (Ref. 4) or $\mathrm{TbAl}_{2},{ }^{9}$ where the lattice strain reaches the value around $1 \%$. Furthermore, x-ray absorption measurements show that the interatomic distances are barely affected by the milling process (see Table I) and an increase in both the lattice strain and the Debye-Waller factor when the milling time increases is observed (see Fig. 9).

As commented previously, the variation of physical parameters is governed by the reduction in the size of the particles and concomitantly by the increase in surface $\mathrm{Yb}^{2+}$ atoms. In fact, from inspection of Fig. 10, it seems that the valence - the value of the magnetic-susceptibility maximaand the high-temperature slope $(S L)$ of $\Delta c$ scale reasonably with $D$. The reduction in $D$ (showing a drastic decrease from bulk to $20 \mathrm{~nm}$ ) and the $D$-independent value of the lattice parameters are especially indicative, in contrast to a less pronounced variation of $\sigma$ and $\eta$ (see Fig. 9) associated with local disorder. Another fact is that the magnetic susceptibility and the specific heat behave differently to the study of chemically substituted series by $\mathrm{Lu}$, where a different situation was found. ${ }^{18}$ One possibility is that the disorder in the chemically substituted system plays a major role, whereas in nanosized $\mathrm{YbAl}_{3}$ series size effects are more relevant as demonstrated above. The solution of this puzzling situation would require the study of nanosized $\mathrm{Yb}_{x} \mathrm{Lu}_{1-x} \mathrm{Al}_{3}$ series.

The driving parameter for the changes of the electronic, magnetic, and thermal properties of $\mathrm{YbAl}_{3}$ milled alloys is the particle surface/volume ratio. The calculation of the fraction of the atoms at the surface results in an increase from $16 \%$ to $30 \%$ when the size of the particles decreases from 20 to $10 \mathrm{~nm}$. In this sense, exhaustive studies on the electronic properties in rare-earth systems have shown that rare-earth atoms with an unstable $f$ shell present a lower valence at the surface than in the bulk of a material. ${ }^{15,32-34}$ In metallic samarium, the spectrum of surface atoms has been resolved by photoemission from both core and valence states. This was demonstrated with Sm metal evaporated into stainless steel and gold plates by tilting the surface normal away from the direction of electron collection, which enhances emission for the surface relative to that from the bulk. The presence of divalent $\mathrm{Sm}$ is clearly detected and explained by a narrowing of the $5 d$ band by virtue of a reduced coordination number, which would raise the Fermi energy and allow the population of the $4 f^{6}$ state. $^{32}$ A similar situation has been found in $\mathrm{YbPd}$ and $\mathrm{Yb}_{3} \mathrm{Pd}_{4}$ compounds by photoemission experiments, where a single divalent surface layer also appears. ${ }^{34}$ An interpretation of the surface valence of rare-earth metals has been given based on the tension force perpendicular to the surface. ${ }^{15}$ With this it is possible to determine the enthalpy $\left(\Delta \mathrm{H}_{\mathrm{II}, \mathrm{III}}\right)$ difference between divalent and trivalent states. For the elements where the difference $-\Delta \mathrm{H}_{\mathrm{II}, \mathrm{III}}$ is below $12 \mathrm{kcal} /$ mol, the surface state will be divalent, which is the case for both $\mathrm{Sm}$ and $\mathrm{Yb}$ atoms. The bonding of trivalent rare-earth atoms is more intense than that of divalent atoms. Therefore, the loss of bonding when a surface is created favors the lower valence state for $\mathrm{Yb}$ (being just the opposite behavior than that observed in some nanosized Ce alloys) ${ }^{11,12}$ where the atoms on the surface present a shift toward a higher valence state, which in this case is the nonmagnetic one [intermediate valence (IV)]. So far, this would indicate that the $\mathrm{YbAl}_{3}$ milled alloys behave similarly to those Ce alloys concerning only the increase in the fraction of nonmagnetic atoms with the reduction in the size of the particles.

\section{CONCLUSIONS}

The influence of the milling process on the IV $\mathrm{YbAl}_{3}$ alloy has been characterized systematically by studying structural, thermal, magnetic, and electronic properties. The structure of this alloy is clearly affected during the milling process and results in a drastic reduction of the particle size to the nanometer scale. In consequence, the milled $\mathrm{YbAl}_{3}$ alloys are formed by a distribution of nanoparticles in which the $\mathrm{Yb}^{2+}$ atomic state is favored at the surface while atoms in deeper layers (in the core) keep the IV as in the unmilled material. Thus, the relation between the size and the fraction of $\mathrm{Yb}$ surface atoms at the interface is the origin of the variations found in the physical properties in the milled $\mathrm{YbAl}_{3}$ alloys. Further investigation in other rare-earth nanoparticle systems is needed to establish more quantitative evidence, for instance, by compositionally diluting the $\mathrm{YbAl}_{3}$ nanoparticles.

\section{ACKNOWLEDGMENTS}

This work was supported by the Direction of the Universities of the Ministry of Science and Education of Spain under Contract No. MAT 2005-06806-c04 and Juan de la Cierva program. X-ray absorption measurements were carried out at beamline BL39XU of Spring-8 facility and beamline BM25A of ESRF experiment number HD-113. We are grateful to G. Castro, M. A. Laguna-Marco, R. Boada, and D. Alba Venero for helping in the experimental work at ESRF. 
${ }^{1}$ Handbook of Magnetism and Advanced Magnetic Materials, edited by Helmut Kronmüller and Stuart Parkin (Wiley, Chichester, 2007), Vol. 1.

${ }^{2}$ J. L. Dormann and D. Fiorani, Magnetic Properties of Fine Particles (North-Holland, London, 1992).

${ }^{3}$ G. Herzer, in Handbook of Magnetic Materials, edited by K. H. J. Buschow (Elsevier Science, Amsterdam, 1998), Vol. 10, p. 415

${ }^{4}$ G. F. Zhou and H. Bakker, Phys. Rev. B 52, 9437 (1995).

${ }^{5}$ I. W. Modder and H. Bakker, Phys. Rev. B 58, 14479 (1998).

${ }^{6}$ I. W. Modder, H. Bakker, and G. F. Zhou, Physica B (Amsterdam) 262, 141 (1999).

${ }^{7}$ D. S. Williams, P. M. Shand, T. M. Pekarek, R. Skomski, V. Petkov, and D. L. Leslie-Pelecky, Phys. Rev. B 68, 214404 (2003).

${ }^{8}$ M. A. Morales, D. S. Williams, P. M. Shand, C. Stark, T. M. Pekarek, L. P. Yue, V. Petkov, and D. L. Leslie-Pelecky, Phys. Rev. B 70, 184407 (2004).

${ }^{9}$ D. P. Rojas, L. Fernández Barquín, J. Rodríguez Fernández, J. I. Espeso, and J. C. Gómez Sal, J. Phys.: Condens. Matter 19, 186214 (2007).

${ }^{10}$ Y. Y. Chen, Y. D. Yao, C. R. Wang, W. H. Li, C. L. Chang, T. K. Lee, T. M. Hong, J. C. Ho, and S. F. Pan, Phys. Rev. Lett. 84, 4990 (2000).

${ }^{11}$ S.-W. Han, C. H. Booth, E. D. Bauer, P. H. Huang, Y. Y. Chen, and J. M. Lawrence, Phys. Rev. Lett. 97, 097204 (2006).

${ }^{12}$ Y. Y. Chen, P. H. Huang, M. N. Ou, C. R. Wang, Y. D. Yao, T. K. Lee, M. Y. Ho, J. M. Lawrence, and C. H. Booth, Phys. Rev. Lett. 98, 157206 (2007).

${ }^{13}$ W. B. Thimm, J. Kroha, and J. von Delft, Phys. Rev. Lett. 82, 2143 (1999).

${ }^{14}$ P. Schlottmann, Phys. Rev. B 65, 024420 (2001).

${ }^{15}$ B. Johansson, Phys. Rev. B 19, 6615 (1979); B. Johansson and N. Martensson, in Handbook on the Physics and Chemistry of Rare Earths, edited by K. A. Gschneidner, Jr., L. Eyring, and S. Hüfner (North-Holland, Amsterdam, 1987), Vol. 10, p. 361.

${ }^{16}$ A. Hiess, J. X. Boucherle, F. Givord, and P. C. Canfield, J. Alloys Compd. 224, 33 (1995).

${ }^{17}$ A. L. Cornelius, J. M. Lawrence, T. Ebihara, P. S. Riseborough, C. H. Booth, M. F. Hundley, P. G. Pagliuso, J. L. Sarrao, J. D. Thompson, M. H. Jung, A. H. Lacerda, and G. H. Kwei, Phys. Rev. Lett. 88, 117201 (2002).

${ }^{18}$ E. D. Bauer, C. H. Booth, J. M. Lawrence, M. F. Hundley, J. L. Sarrao, J. D. Thompson, P. S. Riseborough, and T. Ebihara, Phys. Rev. B 69, 125102 (2004).

${ }^{19}$ T. Ebihara, E. D. Bauer, A. L. Cornelius, J. M. Lawrence, N. Harrison, J. D. Thompson, J. L. Sarrao, M. F. Hundley, and S. Uji, Phys. Rev. Lett. 90, 166404 (2003).

${ }^{20}$ A. C. Hewson, The Kondo Problem to Heavy Fermions (Cambridge University Press, Cambridge, England, 1993).

${ }^{21}$ J. D. Thompson and J. L. Lawrence, in Handbook on the Physics and Chemistry of Rare Earths, edited by K. A. Gschneidner, Jr., L. Eyring, G. H. Lander, and G. R. Choppin (North-Holland,
Amsterdam, 1994), Vol. 19, p. 383.

${ }^{22}$ A. Palenzona, J. Less-Common Met. 29, 289 (1972).

${ }^{23}$ C. Suryanarayana, Prog. Mater. Sci. 46, 1 (2001).

${ }^{24}$ J. Rodriguez-Carvajal, Physica B (Amsterdam) 192, 55 (1993).

${ }^{25}$ J. Rupp and R. Birringer, Phys. Rev. B 36, 7888 (1987).

${ }^{26}$ N. X. Sun and K. Lu, Phys. Rev. B 54, 6058 (1996).

${ }^{27}$ S. Mentese, J.-B. Suck, and V. Reat, Appl. Phys. A: Mater. Sci. Process. A74, 969 (2002).

${ }^{28}$ H. J. Fecht, Phys. Rev. Lett. 65, 610 (1990).

${ }^{29}$ M. Wagner, Phys. Rev. B 45, 635 (1992).

${ }^{30}$ D. Wolf, J. Wang, S. R. Phillpot, and H. Gleiter, Phys. Rev. Lett. 74, 4686 (1995).

${ }^{31}$ P. M. Derlet, R. Meyer, L. J. Lewis, U. Stuhr, and H. Van Swygenhoven, Phys. Rev. Lett. 87, 205501 (2001).

${ }^{32}$ G. K. Wertheim and G. Crecelius, Phys. Rev. Lett. 40, 813 (1978).

${ }^{33}$ J. W. Allen, L. I. Johansson, R. S. Bauer, I. Lindau, and S. B. M. Hagström, Phys. Rev. Lett. 41, 1499 (1978).

${ }^{34}$ M. Domke, C. Laubschat, E. V. Sampathkumaran, M. Prietsch, T. Mandel, G. Kaindl, and H. U. Middelmann, Phys. Rev. B 32, 8002 (1985).

${ }^{35}$ D. C. Koningsberger and R. Prins, X-Ray Absorption: Principles, Applications, Techniques of EXAFS, SEXAFS and XANES, Chemical Analysis (Wiley, New York, 1988).

${ }^{36}$ J. M. Lawrence, G. H. Kwei, P. C. Canfield, J. G. DeWitt, and A. C. Lawson, Phys. Rev. B 49, 1627 (1994).

${ }^{37}$ E. Bauer, Le Tuan, R. Hauser, E. Gratz, T. Holubar, G. Hilscher, H. Michor, W. Perthold, C. Godart, E. Alleno, and K. Hiebl, Phys. Rev. B 52, 4327 (1995).

${ }^{38}$ B. Ravel and M. Newville, J. Synchrotron Radiat. 12, 537 (2005).

${ }^{39}$ A. García Prieto, M. L. Fdez-Gubieda, C. Meneghini, A. GarcíaArribas, and S. Mobilio, Phys. Rev. B 67, 224415 (2003).

${ }^{40}$ L. Del Bianco, F. Boscherini, A. L. Fiorini, M. Tamisari, F. Spizzo, M. V. Antisari, and E. Piscopiello, Phys. Rev. B 77, 094408 (2008).

${ }^{41}$ M. Maurer, J. M. Friedt, and G. Krill, J. Phys. F: Met. Phys. 13, 2389 (1983).

${ }^{42} \mathrm{~J}$. Röhler, in Handbook on the Physics and Chemistry of Rare Earths, edited by K. A. Gschneidner, Jr., L. Eyring, and S. Hüfner (North-Holland, Amsterdam, 1987), Vol. 10, p. 453.

${ }^{43}$ E. Vescovo, L. Braicovich, B. De Michelis, A. Fasana, R. Eggenhöffner, A. Iandelli, G. L. Olcese, and A. Palenzona, Phys. Rev. B 43, 12281 (1991).

${ }^{44}$ C. Dallera, E. Annese, J.-P. Rueff, A. Palenzona, G. Vanko, L. Braicovich, A. Shukla, and M. Grioni, Phys. Rev. B 68, 245114 (2003).

${ }^{45}$ S. N. Mishra, S. Bose, P. Vasa, and P. Ayyub, Phys. Rev. B 71, 094429 (2005).

${ }^{46}$ A. Hiess, J. X. Boucherle, F. Givord, J. Schweizer, E. LelievreBerna, F. Tasset, B. Gillon, and P. C. Canfield, J. Phys.: Condens. Matter 12, 829 (2000). 